\title{
Relación entre estrés laboral y burnout en una muestra de controladores de tráfico aéreo en Chile
}

\section{The relation between work stress and burnout syndrome in a sample of chilean air traffic controllers}

\author{
Carla Aguirre Mas ${ }^{1}$ \\ Agustina Gallo ${ }^{2}$ \\ Alexander Ibarra ${ }^{3}$ \\ José Carlos Sánchez García ${ }^{4}$ \\ 123 Facultad de Psicología, Universidad Católica del Uruguay \\ ${ }^{4}$ Facultad de Psicología Universidad de Salamanca. España
}

\begin{abstract}
Resumen: Esta investigación tiene por objetivo hallar la relación entre el burnout, el estrés laboral, la satisfacción laboral y el conflicto trabajo-familia en controladores de tráfico aéreo, como variables diferenciales: edad, sexo, estado civil, permanencia laboral, tipo de contrato y presencia o ausencia de hijos. Esta investigación es de tipo descriptivo-correlacional de corte transversal. Se aplicaron los Cuestionario: MBI-GS de Burnout, Instrumento de Medición de detonantes de Estrés Laboral para pilotos (IMDELP) y el Cuestionario Conflicto Trabajo-Familia. La muestra fue de 161 personas, mediante muestreo intencionado. Los resultados indican que se obtuvo un nivel moderado de burnout, estrés laboral y conflicto trabajo-familia, existiendo diferencias significativas y directamente proporcionales a la hora de analizar las dimensiones del burnout relacionándolas con extensión de las jornadas de trabajo y agotamiento, agotamiento y conflicto trabajo-familia, estrés laboral y cinismo.
\end{abstract}

Palabras clave: estrés laboral, Burnout, satisfacción laboral, conflicto trabajo-familia, controladores de tráfico aéreo

\begin{abstract}
The purpose of the present study is to identify the relation between burnout syndrome, laboral stress, Labor Satisfaction and conflict work-family, in a sample of Air traffic controllers, taking in consideration the following variables: age, sex, civil status, labor permanence, contract type and presence or absence of children. In this descriptive-correlational study we use the: MBI-GS of Burnout Questionnaire, detonating questionnaire Instrument of measurement of Labor Stress for pilots (IMDELP), and the Conflict Work- Family Questionnaire. The sample was 161 Air Traffic Controllers, through intentional sampling. The results indicate that a moderate level of burnout, work-related stress and work-family conflict. There were significant and directly proportional differences when analyzing the dimensions of burnout, relating them to length of work and exhaustion, exhaustion and conflict work-family, work stress and cynicism.
\end{abstract}

Keywords: work stress, burnout, labor satisfaction, conflict work-family, air traffic controllers

Cómo citar este artículo:

Aguirre Mas, C., Gallo, A., Ibarra, A., Sánchez García, J. C. (2018). Relación entre estrés laboral y burnout en una muestra de controladores de tráfico aéreo en Chile. Ciencias Psicológicas, 12(2), 239-248. doi: https://doi.org/10.22235/cp.v12i2.1688. 


\section{Introducción}

Según Jiménez y Moyano (2008), en ciencias sociales existe un consenso fuertemente asentado de que el trabajo constituye una de las actividades de mayor importancia para la integración y ajuste psicológico y social de los individuos. El trabajo es una necesidad para las personas, pues permite conseguir bienes -directa o indirectamente- y alcanzar compensaciones personales o sociales satisfactorias. Socialmente, el trabajo es considerado como un deber de participación en los esfuerzos de un colectivo, con el fin de contribuir al desarrollo de la sociedad (Moyano \& Ramos, 2007). Así, el trabajo ha ido trascendiendo durante los años desde la esfera de la economía -obtención de bienes- a otros ámbitos relevantes de la vida de las personas -satisfacción y calidad de vida-, generando cambios en las formas de trabajar y nuevos valores sociales. Debido a esto, las empresas están interesadas en generar técnicas que les permita suplir las necesidades personales y promover el bienestar de los trabajadores, generando nuevas políticas en función de mejorar las condiciones para el desempeño de sus trabajadores (Dirección General del Trabajo, 2018).

Peiró y Rodríguez (2008) señalan que los cambios demográficos, los nuevos mercados y las nuevas tecnologías, han generado un gran número de indicadores de absentismo, insatisfacción laboral, accidentes laborales, bajo desempeño y estrés laboral acompañado de burnout.

Según Aguirre (2015) para muestras de pilotos de aeronaves comerciales, es posible conceptualizar cinco fuentes potenciales de estrés en el contexto laboral:

- Condiciones físicas del lugar de trabajo: condiciones propias del diseño y estructura del lugar de trabajo, como ventilación, ruido, iluminación, temperatura, disposición del espacio físico de trabajo, espacios de descanso, higiene, entre otras.

- Relaciones interpersonales: relaciones y contactos establecidos con otras personas en el lugar de trabajo como jerarquías, compañeros de igual o menor jerarquía y relación con los usuarios de los servicios.

- Factores organizacionales: el sistema de reglas que regulan las interacciones y comportamientos del individuo en el trabajo como las políticas, las normativas de horarios y las condiciones para prestaciones.
- Contenidos y demandas del trabajo: como duración excesiva del trabajo, complejidad de las tareas, incapacidad de desarrollar habilidades y los sistemas de turnos.

- Conflicto trabajo-familia o factores extra organizacionales: que son todos aquellos factores de la vida personal que pueden ser potencialmente perjudicados por el trabajo si no interactúan con éste de manera saludable para el trabajador; como, por ejemplo: la posibilidad de estar con su familia. Se ha identificado como estresor con influencia sobre la vida laboral, ante dificultades para balancear las responsabilidades de las esferas laboral y familiar (Otalora, 2007).

En relación a esto, se ha estudiado el estrés laboral y se han propuesto diferentes modelos explicativos, pues diversas condiciones sociales pueden actuar como agentes estresantes y modificar la salud de las personas (Peiró \& Rodríguez, 2008; Sadin, 2003). Asimismo, se han estudiado los efectos sobre los desencadenantes de estrés, que no sólo se limitan a variables sociodemográficas como la edad, el nivel de ingresos, el sexo, entre otras (Kanner et al., 1981; Fernández \& Piñol, 2000), sino que incluyen otras variables cotidianas donde el trabajador experimenta vivencias negativas asociadas al contexto laboral.

Los períodos repetidos de estrés suelen preceder al síndrome de burnout, traducido como quemarse por el trabajo, que resulta de la interacción de variables del entorno laboral y personal aludiendo a una serie de síntomas y sentimientos asociados al estrés laboral. Estos síntomas se caracterizan por un agotamiento emocional, falta de energía, distanciamiento y cinismo hacia los destinatarios, sentimientos de incompetencia, deterioro del autoconcepto profesional, actitudes de rechazo hacia el trabajo (Gil-Monte \& Peiró, 1999; Jiménez \& Moyano, 2008; Olmedo et al., 2001); otros diversos síntomas psicológicos como irritabilidad, ansiedad, tristeza y baja autoestima e insatisfacción laboral (Moriana, 2006) y síntomas físicos como enfermedades respiratorias y alergias (Fu, Lindgren, Wieslander, Janson, \& Norbäck, 2016).

Maslach y Jackson (1986) propusieron tres dimensiones del burnout:

- Agotamiento emocional: son las sensaciones de esfuerzo físico y hastío emocional que se producen como consecuencia de las continuas interacciones que los trabajadores deben mantener entre ellos. 
- Cinismo o despersonalización: supone el desarrollo de actitudes y respuestas cínicas hacia las personas a quienes los trabajadores prestan sus servicios, acompañado de un incremento de irritabilidad, donde el trabajador se distancia de las personas con los que trabaja y de los usuarios de los servicios, tratando de hacerles culpables de sus frustraciones y problemas laborales.

- Baja eficacia personal: pérdida de confianza en la realización personal y la presencia de un negativo autoconcepto como resultado de las situaciones ingratas. Sentimiento de falta de competencia, de esfuerzo o conocimiento, carencia de expectativas, de horizontes laborales y una insatisfacción generalizada. Dando como consecuencia: impuntualidad, abundancia de interrupciones, evitación del trabajo, absentismo y abandono de la profesión.

El estrés laboral y el burnout no sólo desencadenan problemas en el ámbito de la salud mental/física, de los individuos/familia, sino que también tiene repercusiones económicas para las organizaciones. Se ha encontrado relación entre ausentismo, rotaciones y la productividad laboral con problemas de salud prevenibles, relacionados al estrés laboral (Guic, Bilbao, \& Bertin, 2002).

La satisfacción laboral hace alusión a la calidad de vida laboral en relación al compromiso organizacional y elementos del trabajo tales como las condiciones ambientales, salario, seguridad laboral, posibilidades de promoción y las tareas realizadas (Segurado \& Argullo, 2002).

Sánchez y Quiroga (1995) estudiaron a tres tipos de parejas: tradicionales, con doble ingreso y con doble carrera. Encontraron mayor satisfacción en las trabajadoras solteras de mayor nivel educativo. También en las mujeres predomina la congruencia entre ambos mundos: hay satisfacción o insatisfacción en ambos; mientras que en los hombres es más frecuente la incongruencia: hay satisfacción en un entorno y no en el otro.

Al evaluar riesgos, los profesionales del medio aeronáutico ocupan el tercer lugar a nivel mundial entre las profesiones con mayor exposición a estrés, debido a la exposición física de: afecciones músculo-esqueléticas, auditivas, entre otras o a las exigencias psicológicas: ansiedad, depresión, ideación suicida, entre otras (Aguirre et al, 2015; Wu et al., 2016).

Los controladores de tráfico aéreo (CTA) en Chile son el personal que está encargado de dirigir el tránsito de aeronaves en el espacio aéreo y en los aeropuertos de un modo seguro, ordenado y rápido, autorizando a los pilotos de los aviones con instrucciones y proveyendo información dentro del espacio aéreo bajo su responsabilidad, con el objeto de prevenir colisiones entre aeronaves y obstáculos en el área de maniobras de los aeródromos y aeropuertos. Su labor es crítica puesto que están a cargo de la vida de varias personas que viajan en cada vuelo, preocupándose, además, de los posibles cambios meteorológicos y otros imprevistos. Todo esto genera tensión constante, además de trabajar en las condiciones menos apropiadas con exposición a ruido, calor, entre otros factores (Miranda, 2015).

La presente investigación pretende identificar si existe relación entre el estrés laboral y la percepción de burnout, con las posibles implicaciones que estos vínculos suponen para la calidad de vida de los CTA de Chile.

\section{Método}

\section{Procedimiento}

El estudio fue transversal, con el burnout, los estresores laborales y la satisfacción laboral en los controladores de tráfico como variables del estudio. Se incluyó, además: el estado civil, la edad, el sexo, la presencia de hijos, la pertenencia laboral y el tipo de contrato. Se utilizó una sola medición muestral con tasa de respuesta al 10\% del total de CTA contactados. Se calcularon los estadísticos descriptivos univariados y bivariados mediante el programa estadístico SPSS 22.0. Se destaca que para la recolección de los datos se solicitó a los participantes la firma de consentimiento informado en cada escala y la aprobación de la investigación se dio en el marco de la revisión de la Comisión de Ética de la Facultad de Psicología de la Universidad de Salamanca, España.

\section{Participantes}

La muestra estuvo compuesta por 161 CTA de entre 30 a 64 años de edad. La técnica de muestreo fue no probabilística y por conveniencia. La siguiente tabla recoge los datos sociodemográficos característicos de la muestra (tabla 1). 
Tabla 1

Datos sociodemográficos

\begin{tabular}{|c|c|c|c|}
\hline Datos & Distribución & Frecuencia & Porcentaje \\
\hline \multirow[t]{3}{*}{ Sexo } & Hombre & 131 & 81.4 \\
\hline & Mujer & 30 & 18.6 \\
\hline & Total & 161 & 100.0 \\
\hline \multirow[t]{6}{*}{ Edad } & $=30$ & 21 & 13.0 \\
\hline & $31-41$ & 63 & 39.1 \\
\hline & $42-52$ & 46 & 28.6 \\
\hline & $53-63$ & 26 & 16.1 \\
\hline & $=64$ & 5 & 3.1 \\
\hline & Total & 161 & 100.0 \\
\hline \multirow[t]{7}{*}{ Estado Civil } & Soltero & 34 & 21.1 \\
\hline & Casado & 111 & 68.9 \\
\hline & Separado & 9 & 5.6 \\
\hline & Divorciado & 5 & 3.1 \\
\hline & Viudo & 1 & 0.6 \\
\hline & Otro & 1 & 0.6 \\
\hline & Total & 161 & 100.0 \\
\hline \multirow[t]{4}{*}{ Hijos } & $\mathrm{Si}$ & 130 & 80.7 \\
\hline & No & 30 & 18.6 \\
\hline & Perdidos sistema & 1 & 0.6 \\
\hline & Total & 161 & 100.0 \\
\hline \multirow{6}{*}{$\underset{\text { trabajo }}{\text { A re a }}$ de } & Torre de control & 32 & 19.9 \\
\hline & Oficina de control de aproximación & 30 & 18.6 \\
\hline & Centro de control & 71 & 44.1 \\
\hline & Otro & 22 & 13.7 \\
\hline & Perdidos Sistema & 6 & 3.7 \\
\hline & Total & 161 & 100.0 \\
\hline \multirow{4}{*}{$\begin{array}{l}\text { T i p o d e } \\
\text { contrato }\end{array}$} & Fijo & 109 & 67.7 \\
\hline & Temporal & 49 & 30.4 \\
\hline & Perdidos Sistema & 3 & 98.1 \\
\hline & Total & 161 & 100.0 \\
\hline Permanencia & $=5$ años & 23 & 14.3 \\
\hline \multirow[t]{5}{*}{ laboral } & 6 A 11 años & 23 & 14.3 \\
\hline & 12 A 17 años & 35 & 21.7 \\
\hline & 18 A 23 años & 34 & 21.1 \\
\hline & $=24$ años & 46 & 28.6 \\
\hline & Total & 161 & 100.0 \\
\hline \multirow{2}{*}{$\begin{array}{l}\text { Lugar de } \\
\text { formación }\end{array}$} & Escuela Técnica Aeronáutica & 161 & 100.0 \\
\hline & Total & 161 & 100.0 \\
\hline
\end{tabular}

\section{Instrumentos}

- Cuestionario (A) MBI-GS (Schaufeli, Leiter, Maslach, \& Jackson, 1996). Este instrumento evalúa el burnout, está compuesto por 15 ítems separados en 3 dimensiones: agotamiento, despersonalización y eficacia personal. Los ítems proponen afirmaciones de las actitudes y emociones personales de los controladores sobre su desempeño y de su trabajo en general, los que son respondidos en forma de escala Likert de 6 puntos que va de nunca (1) a siempre (6). La fiabilidad de la totalidad de la escala de burnout en esta muestra de CTA, medida por el coeficiente de consistencia interna alpha de Cronbach, es adecuada $(\alpha=.849)$.
- Instrumento de medición de detonantes de Estrés Laboral para pilotos (IMDELP) (Aguirre et al., 2015). Evalúa las cinco dimensiones de estresores descritas en la teoría: condiciones físicas, relaciones interpersonales, factores organizacionales, factores extra organizacionales y contenidos $\mathrm{y}$ demandas del trabajo. Si bien es un instrumento creado para evaluar detonantes de estrés laboral en pilotos de aeronaves comerciales, se propuso adecuar los ítems a las características de la muestra. Las respuestas se recogieron a partir de escala tipo likert de 5 puntos los que van de (1) "muy en desacuerdo" a (5) "muy de acuerdo". La fiabilidad de la escala se estimó por medio del coeficiente 
de consistencia interna de alfa de Cronbach, en la muestra de CTA fue de un $\alpha$ igual a .927.

- Satisfacción laboral (Aguirre et al., 2015). Compuesto por 20 ítems con frases afirmativas adaptadas a las características de la muestra, con cinco alternativas de respuestas en una escala tipo lickert de 5 puntos que va desde "muy insatisfecho" (1) a "muy satisfecho" (5). Esta escala está compuesta por las dimensiones: satisfacción con la supervisión, con el trabajo, con el ambiente físico, con las prestaciones, y con las oportunidades de participación.

- Percepción de desempeño (Aguirre et al., 2015). Es una escala unidimensional de 2 ítems, construido y validado por Aguirre (2006) y adaptado a las características de la presente muestra. El formato de respuesta es mediante una escala likert de 5 puntos que va de "muy de acuerdo" (1) a "muy en desacuerdo" (5).

- Síntomas psicosomáticos de estrés (Aguirre et al., 2015). Para hacer una evaluación de la activación fisiológica de las respuestas de estrés se usó un cuestionario validado en estudios anteriores para muestras de pilotos de aeronaves comerciales (Aguirre, 2015), al cual se le adecuaron los ítems según las características de esta muestra. Consta de diez ítems con frases afirmativas relativas a la percepción del individuo de los síntomas de estrés. Tales ítems deben responderse con una sola alternativa de respuesta en una escala likert de 5 puntos, respuesta que van de "muy de acuerdo" (1) a "muy en desacuerdo" (5).

- Cuestionario (C) Conflicto Trabajo Familia, desarrollado por Carlson, Kacmar y Williams (2000), se usa para medir los tipos de conflicto trabajo-familia en base al estrés, al comportamiento y al tiempo, además cuenta con dos direcciones en las que se da el conflicto de trabajo a familia y de familia a trabajo, con una confiabilidad de 0,927 . La escala es de tipo likert de 1 a 5 con 18 afirmaciones.

Los puntos de corte se efectuaron mediante intervalos de confianza, que se distribuyeron de la siguiente forma a) Alto, b) Bajo, c) Crítico, técnica diseñada por Vauro (2014) y Aguirre (2015), para la proyección de dichos valores, los mismos tuvieron como fuente, la mediana de cada intervalo.

\section{Resultados}

En los niveles de burnout, prevalece un bajo nivel, seguido por un alto grado de afectación y por niveles críticos (tabla 2). Esta última categoría se relaciona con altos niveles de riesgo para experimentar sintomatología física y psicológica relacionada con el burnout.

Tabla 2
MBI-GS porcentaje de incidencia de
burnout para la muestra
\begin{tabular}{lc} 
Nivel de Burnout & Porcentaje \\
\hline Bajo & 44.2 \\
Alto & 27.3 \\
Crítico & 26.1 \\
Valores perdidos & 2.4
\end{tabular}

Respecto a las dimensiones de la escala MBI-GS Burnout, puede notarse en la tabla 3 que la eficacia personal es la que presenta mayores niveles, seguido por el agotamiento emocional y en menores niveles el cinismo.

Tabla 3

Porcentaje de incidencia para las dimensiones de burnout

\begin{tabular}{lccc}
\hline Nivel & $\begin{array}{c}\text { Agotamiento } \\
\text { emocional }\end{array}$ & Cinismo & $\begin{array}{c}\text { Eficacia } \\
\text { personal }\end{array}$ \\
\hline Muy bajo & 32.3 & 50.3 & 1.9 \\
Bajo & 25.5 & 22.4 & 5 \\
Moderado & 31.1 & 13.7 & 10.6 \\
Alto & 3.1 & 6.2 & 29.8 \\
Muy alto & 11.2 & 3.7 & 50.9 \\
\hline
\end{tabular}

En cuanto a los niveles de estrés laboral, los niveles más altos se encontraron en la dimensión contenido y demandas del trabajo, seguido por los factores extra organizacionales. Los controladores reportaron como menos estresante las condiciones físicas del trabajo y el clima interpersonal (tabla 4).

Tabla 4

Niveles de estrés laboral por dimensión (porcentajes)

\begin{tabular}{lccc}
\hline Dimensión & Bajo & Moderado & Alto \\
\hline Condiciones físicas & 63.4 & 29.8 & 6.8 \\
Relaciones interpersonales & 52.2 & 44.1 & 3.7 \\
Factores organizacionales & 41 & 46.6 & 12.4 \\
Factores extra & 49.1 & 37.3 & 13.7 \\
organizacionales & 6.2 & 32.2 & 61.5 \\
\hline
\end{tabular}

Los controladores reportaron mejor grado de satisfacción con su trabajo y con la supervisión. Mientras que las prestaciones como el sueldo y demás remuneraciones, las opciones de participación y el ambiente físico son las que presentan menor grado de satisfacción (tabla 5). 
Tabla 5

Niveles de satisfacción por dimensión (porcentajes)

\begin{tabular}{lccccc}
\hline Nivel & Supervisión & Prestaciones & Trabajo & Participación & $\begin{array}{c}\text { Ambiente } \\
\text { físico }\end{array}$ \\
\hline Bajo & 23 & 43.5 & 16.2 & 43.5 & 42.9 \\
Moderado & 49.1 & 40.4 & 47.2 & 31.1 & 46 \\
Alto & 28 & 16.1 & 36 & 25.5 & 11.2 \\
\hline
\end{tabular}

En cuanto al desempeño laboral percibido, solo un $3.1 \%$ de la muestra considera que su desempeño en el contexto laboral es bajo; el 16.8\% lo percibe en un nivel moderado y el $80.1 \%$ manifiesta tener un alto desempeño laboral.

En relación con la dimensión síntomas psicosomáticos de estrés, el $88.2 \%$ de la muestra presenta una baja percepción de estrés; el 10.6\% manifiesta una percepción moderada de estrés y solo el $1.2 \%$ percibe estar estresado.

En la tabla 6 se muestra el nivel de conflicto trabajo-familia con base a sus dimensiones y las dos direcciones en las que se da: conflicto de trabajo hacia la familia y de la familia al trabajo. En general, reportaron moderados niveles de conflicto en el estrés asociado a cada rol, en los comportamientos y en las demandas temporales, al igual que con el grado de interferencia del trabajo en la dinámica familiar.

Se encontraron diferencias significativas en la dimensión agotamiento según el rango de edad $(F=2.989 ; p=.021)$. Los controladores aéreos en el rango de edad de 53 a 63 años reportan menor grado de agotamiento que los menores a 53 o mayores a $63(F=4.408 ; p=.044)$. No se hallaron diferencias en cinismo ni en eficacia personal según la edad ( $\mathrm{p}>.05)$.

También se hallaron diferencias significativas según el sexo: las mujeres tienden a percibir más agotamiento que los hombres $(F=4.408 ; p=.044)$. No se hallaron estas diferencias según el sexo en las dimensiones cinismo y eficacia personal $(p>.05)$.

Respecto al estado civil, a la presencia o ausencia de hijos y al tipo de contrato laboral, no presentaron relación significativa con ninguna de las dimensiones del burnout, pero sí lo hizo la variable permanencia laboral, aunque sólo con la dimensión agotamiento, resultando ser estadísticamente significativo $(F=2.934 ; p=.019)$. Si bien todos los controladores aéreos señalaron percibir bajos niveles de agotamiento, aquellos con 5 años de experiencia laboral son lo que puntúan más alto en la dimensión de agotamiento.

Si hallaron diferencias estadísticamente significativas $(p<.05)$ según la edad en tres dimensiones del estrés laboral: en condiciones físicas, en factores extra organizacionales y en contenidos y demandas. Los controladores del grupo etario de 64 años reportan más bajo estrés que el resto de los grupos, con relación a las condiciones físicas y a los factores extra organizacionales. Mientras que el grupo con edades entre 53 y 63 reportó menor nivel de estrés relacionado con los contenidos y las demandas.

Los controladores del grupo estado civil "viudos" puntuaron más alto al identificar los contenidos y demandas del trabajo como estresores de moderado, en comparación con el resto de los grupos, que puntuaron bajo nivel de estrés $(p<.05)$. No se hallaron diferencias según el estado civil, en el resto de las dimensiones de estrés laboral.

Los controladores sin hijos identifican más estrés laboral relacionado con el ambiente físico, aunque tanto estos como aquellos con hijos no perciben que el ambiente físico sea un detonante significativo $(p>.05)$. No se hallaron diferencias en el resto de las dimensiones de estrés laboral según la presencia o no de hijos.

El grupo de controladores con contrato fijo identificó más estrés con relación al ambiente físico, en comparación con el de contrato temporal $(F=4.630 ; p=.033)$; aunque ambos grupos ubican bajo nivel de estrés relacionado con este factor. No

Tabla 6

Niveles de conflicto trabajo-familia por dimensiones

\begin{tabular}{lcccc}
\hline Nivel & Estrés & Comportamiento & Tiempo & $\begin{array}{c}\text { Interferencia del } \\
\text { trabajo en la familia }\end{array}$ \\
\hline Bajo & 31.7 & 27.3 & 29.2 & 27.2 \\
Moderado & 66.5 & 67.7 & 67.1 & 67.1 \\
Alto & 0.6 & 3.1 & 2.5 & 2.5 \\
\hline
\end{tabular}


se hallaron diferencias estadísticamente significativas según el tipo de contrato, en el resto de las dimensiones del estrés laboral.

Los controladores con más tiempo de trabajo puntúan más bajo que los de menor tiempo de permanencia laboral en la identificación de los contenidos y las demandas laborales como estresores $(F=2,523 ; p=.043)$; aunque todos identifican bajo nivel de estrés relacionado con este factor. No se hallaron diferencias estadísticamente significativas en el resto de dimensiones del estrés laboral, según la permanencia laboral.

En cuanto a la relación entre satisfacción laboral y edad, solo el grupo con mayor edad $(=64)$ puntúa más alto en todas las dimensiones de satisfacción $(F=1.801 ; p=.131)$.

Tanto los hombres como las mujeres incluidos en la muestra perciben bajos niveles de satisfacción frente al trabajo, aunque las mujeres tienen una percepción más favorable $(p<.05)$; no así con la dimensión satisfacción con el ambiente físico, en la cual puntúan más alto los hombres $(p<.05)$. No se hallaron diferencias estadísticamente significativas en el resto de dimensiones de satisfacción según el sexo.

Los controladores con hijos tienen una percepción más favorable del ambiente físico que aquellos sin hijos $(F=1.801 ; p=.131)$. No se hallaron diferencias significativas según la presencia de hijos con el resto de las dimensiones de satisfacción.

Se hallaron diferencias estadísticamente significativas según la permanencia laboral con el grado de satisfacción en sus diferentes dimensiones $(p<.05)$. Pero no se hallaron diferencias en el grado de satisfacción laboral según el tipo de contrato o según el estado civil $(p<.05)$. Tampoco se encontró relación entre el estrés laboral, el desempeño y las variables sociodemográficas incluidas en el estudio $(p<.05)$.

Por otro lado, se encontró que los contenidos y las demandas del trabajo no tienen una relación estadísticamente significativa con ninguna de las dimensiones del burnout $(p>.05)$.

Se encontró que a mayor percepción favorable respecto a las relaciones interpersonales que se dan en el trabajo, disminuye la percepción de agotamiento en los controladores de la muestra y viceversa. Por el contrario, aumenta el reporte de agotamiento a medida que aumentan los estresores laborales relacionados con el ambiente físico, con los factores organizacionales y con los extra organizacionales (tabla 7).

Tabla 7

Correlaciones entre agotamiento y dimensiones de estrés laboral

\begin{tabular}{lcc}
\multicolumn{1}{c}{$\begin{array}{c}\text { Dimensiones } \\
\text { Estrés laboral }\end{array}$} & Correlación & $\begin{array}{c}\text { Significancia } \\
p\end{array}$ \\
\hline Relaciones interpersonales & -.259 & .001 \\
Ambiente físico & .447 & .000 \\
Factores organizacionales & .266 & .001 \\
Factores extra organizacionales & .430 & .000 \\
\hline
\end{tabular}

A medida que aumenta la percepción favorable de las relaciones interpersonales en el trabajo, disminuye la percepción de cinismo y viceversa. La relación es positiva entre el cinismo y el ambiente físico, los factores organizacionales y los extra organizacionales como factores desencadenantes del estés laboral (tabla 8).

Tabla 8

Correlaciones entre cinismo y dimensiones de estrés laboral

\begin{tabular}{lcc} 
Dimensiones & Correlación & \multicolumn{2}{l}{ Significancia } \\
Estrés laboral & \multicolumn{2}{c}{$p$} \\
Relaciones interpersonales & -.321 & .000 \\
Ambiente físico & .250 & .002 \\
Factores organizacionales & .416 & .000 \\
Factores extra organizacionales & .427 & .000 \\
\hline
\end{tabular}

Al aumentar la percepción de estrés laboral según las relaciones interpersonales, también aumenta el reporte de eficacia personal, mientras que el ambiente físico, los factores organizacionales y los extra organizacionales presentan una asociación negativa con la eficacia personal (tabla 9).

Tabla 9

Correlaciones entre eficacia personal y dimensiones de estrés laboral

\begin{tabular}{lcc}
\hline Dimensiones & \multirow{2}{*}{ Correlación } & \multicolumn{2}{l}{ Significancia } \\
Estrés laboral & $p$ & \\
\hline Relaciones interpersonales & .224 & .005 \\
Ambiente físico & -.182 & .022 \\
Factores organizacionales & -.227 & .000 \\
Factores extra organizacionales & -.182 & .022 \\
\hline
\end{tabular}

A mayor agotamiento menor satisfacción laboral y viceversa; y a mayor cinismo menor satisfacción laboral y viceversa. Ocurre lo contrario con la eficacia personal, mientras mayor es la satisfacción laboral también será mayor la eficacia personal y viceversa (tabla 10). 
Tabla 10

Correlaciones entre satisfacción laboral y burnout

\begin{tabular}{lcccccc}
\hline \multirow{2}{*}{$\begin{array}{c}\text { Dimensiones } \\
\text { Satisfacción laboral }\end{array}$} & \multicolumn{5}{c}{ Dimensiones de burnout } \\
\cline { 2 - 7 } & \multicolumn{2}{c}{ Agotamiento } & \multicolumn{2}{c}{ Cinismo } & \multicolumn{2}{c}{ Eficacia personal } \\
\hline Supervisión & $r$ & $p$ & $r$ & $p$ & $r$ & $p$ \\
Prestaciones & -.360 & .000 & -.309 & .000 & .244 & .002 \\
Trabajo & .253 & .001 & -.301 & .000 & .248 & .002 \\
Opciones de participación & -.337 & .000 & -.399 & .000 & .223 & .005 \\
Ambiente físico & -.198 & .012 & -.274 & .001 & .195 & .014 \\
& -.258 & .001 & -.247 & .002 & & $p>.05$ \\
\hline
\end{tabular}

A mayor grado de agotamiento y cinismo, mayor es el reporte de síntomas psicosomáticos de estrés $(r=.357 ; p=.000$ y $r=.257 ; p=.001$ respectivamente). A la inversa, a mayor percepción de síntomas psicosomáticos de estrés, menor grado de eficacia personal $(r=-.163 ; p=.041)$.

A menor grado de agotamiento y cinismo, mayor grado de desempeño $(r=-.282 ; p=.000$ y $r=$ $-.233 ; p=.004$ respectivamente). Por el contrario, a mayor percepción de desempeño, mayor grado de eficacia personal $(r=.241 ; p=.002)$.

En la medida que se identifican los factores del ambiente físico como estresantes, también aumenta la percepción de conflicto entre el trabajo y la familia $(r=.370 ; p=.000)$.

Además, se encontró que en la medida que se identifican a los factores organizacionales y a los extra organizacionales como desencadenantes del estrés, también aumenta la percepción de mayor conflicto trabajo-familia $(r=.397 ; p=.000$ y $r=$ $.604 ; p=.000$, respectivamente).

Por otra parte, a mayor percepción favorable respecto a las relaciones interpersonales que se dan en el trabajo, disminuye la percepción de conflicto trabajo-familia $(r=-0.258 ; p=.001)$.

Solo el factor contenidos y demandas del trabajo no se relaciona significativamente con agotamiento $(p>.05)$.

\section{Conclusión}

La mayoría de los CTA, perciben niveles críticos del síndrome de burnout, sintiéndose moderadamente agotados y generando un distanciamiento emocional entre compañeros de trabajo. Esto se condice con la teoría, al aludir que el estrés laboral crónico o burnout, es una de las principales fuentes de riesgo psicosocial en el trabajo (Bausela, 2005; Olmedo et al., 2001).

Más de la mitad de la muestra señala que las relaciones interpersonales son de alta calidad, siendo un factor protector de estrés laboral (Peiró, 1999; Díaz et al., 2001). Son bajas las condiciones negativas organizacionales, los controladores encuentran que la empresa brinda apoyo y favorece el desarrollo de sus competencias. Dado lo anterior, cabe esperar una mejora en la satisfacción laboral (López, Osca, \& Peiró, 2007).

Los contenidos y demandas propias del trabajo como controlador aéreo sí son percibidos como generadores de estrés; lo que es congruente con las exigencias y responsabilidades de los controladores (Miranda, 2008).

Se observó que la mayoría de los controladores de la muestra considera que existe un nivel de conflicto moderado-bajo, respecto a compatibilizar el tiempo que se dispone para el trabajo y la familia; lo que implica menor incidencia de problemas que se trasfieran del área laboral al familiar, y viceversa (Caviglia, 2016). Dado este resultado, también se esperaría niveles altos de satisfacción laboral, lo cual fue congruente con los datos, salvo en lo referente a las prestaciones.

Los controladores participantes del estudio reportaron niveles moderados a altos de desempeño laboral, lo que reduce las pérdidas económicas relacionadas con el estrés laboral, que están por 1 orden del 40\% (Guic, Bilbao, \& Bertin, 2002).

Contrario a lo esperado en los CTA Chile, no se encontró un nivel alto de síntomas psicosomáticos de estrés; lo que podría relacionarse con factores protectores como el balance entre las demandas familiares y laborales (Jiménez \& Moyano, 2008).

Según los resultados, entre los factores que podrían proteger a los CTA de experimentar burnout están: las relaciones interpersonales dentro de la organización, la satisfacción laboral y el desempeño. Estos factores podrían reducir el riesgo de experimentar distanciamiento emocional, agotamiento y síntomas psicosomáticos relacionados con el estrés; por medio de un aumento de la 
sensación de eficacia personal para afrontar estas demandas. Por el contrario, los factores relacionados con el ambiente físico, los organizacionales y los extra organizacionales, podrían ser generadores de burnout.

Según los resultados, al aumentar la edad parece pesar más el estrés debido a los contenidos y demandas y disminuye la relevancia del ambiente físico. Además, a menor edad y mayor antigüedad, habrá menores niveles de agotamiento. Se sabe que entre más tiempo de permanencia en un empleo demandante, mayor estrés se experimentará (Serrano, 2001). Servirán como factores protectores una mayor permanencia laboral y el tener estabilidad laboral, que se agrega a la satisfacción, el desempeño y las relaciones interpersonales.

Las mujeres tienen una percepción más favorable del trabajo, pero menor respecto al ambiente físico, en la cual puntúan más alto los hombres. Es posible que esta diferencia tenga que ver con la valoración positiva de las relaciones interpersonales, en dónde las mujeres tienden a puntuar más alto (Ortega \& Revilla, 2008).

Los CTA con hijos tienen una percepción más favorable del ambiente físico del trabajo y se muestran con mayor satisfacción que aquellos sin hijos. Este resultado fue contrario lo esperado (Cifre \& Salanova, 2004), quizá debido al uso de recursos para balancear las demandas familiares con las laborales.

Por el último, los CTA viudos tienden a reportar mayor estrés, lo que se relaciona con menor disponibilidad de recursos de apoyo que podrían amortiguar las exigencias laborales.

\section{Referencias}

Aguirre, C. (2006). De los estresores laborales al estrés. Mach 82. Revista de SEPLA, 126, 20-23.

Aguirre, C. (2015). Estresores Laborales y Calidad de Vida en Pilotos Hispanoparlantes de Aeronaves Comerciales. (Tesis Doctoral). Universidad de Salamanca. España.

Bausela E. (2005). El síndrome de quemarse por el trabajo (burnout) de Gil- Monte. Anales de psicología, 21(2), 345-346.

Carlson, D., Kacmar, K., \& Williamns, L. (2000). Construction and initial validation of a multidimensional measure of work-family conflict. Journal of Vocational Behavior, 56, 249-276. doi: https://doi.org/10.1006/ jvbe.1999.1713

Caviglia, A. (2016). Trabajo y familia: roles complementarios. Recuperado de http://www.robertohevens.jazztel.es/ Trabajo\%20y\%20Familia.pps

Cifre, E. \& Salanova, M. (2004). Estrategias de conciliación familia/trabajo: buscando la calidad de vida. Estudios financieros, 259, 111-154.
Díaz, R. Latigue, T. , \& Acosta, M. (2001). Síndrome de burnout: desgaste emocional en cirujanos dentistas. Revista $A D M$, 53(2), 63-67.

Dirección General del Trabajo, Chile. (2018). Recuperado de http://www.dt.gob.cl/portal/1626/w3-channel.html

Fernández, J. \& Piñol, E. (2000). Horario laboral y salud: consecuencias psicológicas de los turnos de trabajo. Revista de Psicopatología y Psicología Clínica, 5, 207 222. doi: 10.5944/rppc.vol.5.num.3.2000.3899

Fu, X., Lindgren, T., Wieslander, G., Janson, C., \& Norbäck, D. (2016). Respiratory illness and allergy related to work and home environment among commercial pilots. PLoS ONE, 11(10), 1-18. doi: https://doi.org/10.1371/ journal.pone.0164954

Gil-Monte, P. \& Peiro, J. (1999). Perspectivas teóricas y modelos interpretativos para el estudio del síndrome de quemarse por el trabajo. Anales de psicología. 15(2), $261-268$.

Guic, E., Bilbao, M., \& Bertin, C. (2002). Estrés laboral y salud en una muestra de ejecutivos chilenos. Revista médica de Chile, 130(10), 1101-1112. doi: http://dx.doi. org/10.4067/S0034-98872002001000004

Jiménez, A. \& Moyano, E. (2008). Factores Laborales de equilibrio entre trabajo y familia medios para mejorar la calidad de vida. Revista universum, 23,1 - 18. doi: http://dx.doi.org/10.4067/S0718-23762008000100007

Kanner, A., Coyne, C., Schaefer, C., \& Lazarus, R. (1981). Comparison of two modes of stress measurement: Daily hassles and uplifts versus major life events. Journal of behavioral medicine, 4(1), 1-39.

López, B., Osca, A., \& Peiro, J. (2007). El papel modulador de la implicación con el trabajo en la relación entre el estrés y la satisfacción laboral. Psicothema, 19(1), 81-87.

Maslach Burnout Inventory Manual (1996). Palo Alto, California: Consulting Psychologists Press, 19-26.

Maslach, C. \& Jackson, S. (1986). The measurement of experinciend bournout. Journal of Ocupational Behavior, 2, 99-113.

Miranda, P. (2015). Gestión de los recursos aeroportuarios. Memoria para optar al título de Gestión Aeronaútica. Universidad Autónoma de Barcelona.

Moriana, J. (2006). Burnout y cuidado de la salud de los voluntarios. Revista electrónica de intervención psicosocial y psicología comunitaria, 1(2), 52-57.

Moyano Díaz, E., \& Ramos Alvarado, N. (2007). Bienestar subjetivo: midiendo satisfacción vital, felicidad y salud en población chilena de la Región Maule. Universum (Talca), 22(2), 177-193. doi: http://dx.doi.org/10.4067/ S0718-23762007000200012

Olmedo, M. Santed, M. Jiménez, R., \& Gómez, M. (2001). El síndrome del burnout: variables laborales personales y psicopatológicas asociadas. Psiquis, 22(3), 117-129.

Ortega, C. \& Revilla, M. (2008). Cultura trabajo-familia, calidad de vida profesional y satisfacción laboral en una muestra de tripulantes de cabina de Lan Chile S.A. (Tesis de Licenciatura). Universidad de Talca. Chile.

Otalora, G. (2007). La relación existente entre el conflicto trabajo familia y el estrés individual en dos organizaciones colombianas. Revista Cuaderno de Administración, 22(24) 1- 21.

Peiro, J. (1999). El estrés laboral una perspectiva individual y colectiva. Revista de Psicología del trabajo y de las Organizaciones, 15(2), 267-314.

Peiro, J. \& Rodríguez, I. (2008). Estrés laboral, liderazgo y salud organizacional. Papeles del psicólogo, 29(1), 68-82.

Sadin, B. (2003). El estrés: un análisis basado en el papel de los factores sociales. Revista internacional de psicología clínica y de la salud, 3(1), 141-157. 
Sánchez, M. \& Quiroga, M. (1995). Relaciones entre satisfacción familiar y laboral: variables moduladoras. Anales de psicología, 11(1), 63-75.

Segurado, A. \& Argullo, E. (2002). Calidad de vida laboral: hacia un enfoque integrador desde la psicología social. Psicothema, 14(4), 828 - 836.

Serrano, C. (2001). Psicología en Aviación. Conceptos Básicos de Fisiología de Aviación, 1-5. España

Schaufeli W., Leiter M., Maslach, C., \& Jackson S. (1996). The Maslach Burnout Inventory: General Survey (MBI-GS). En: Maslach C, Jackson SE, Leiter MP, ed.
Vauro, M. (2014). El Estado del contrato psicológico y su relación con la calidad de vida laboral en profesionales de salud pública de Chile. (Tesis doctoral). Universidad de Salamanca, España.

Wu, A., Donnelly-McLay, D., Weisskopf, M., McNeely, E., Betancourt, T., \& Allen, J. (2016). Airplane pilot mental health and suicidal thoughts: a cross-sectional descriptive study via anonymous web-based survey. Environmental Health, 15-121. doi: 10.1186/s12940016-0200-6 